Revue des patrimoines

12 | 2009

Le patrimoine religieuX des $\mathrm{XIX}^{\mathrm{e}}$ et $\mathrm{XX} \mathrm{X}^{\mathrm{e}}$ siècles $-2^{\mathrm{e}}$ partie

\title{
Les objets mobiliers des XIXe et XXe siècles : les
} sources

\section{Laurence de Finance}

\section{(2) OpenEdition}

\section{Journals}

Édition électronique

URL : http://journals.openedition.org/insitu/4863

DOI : $10.4000 /$ insitu.4863

ISSN : 1630-7305

Éditeur

Ministère de la Culture

Référence électronique

Laurence de Finance, "Les objets mobiliers des XIXe et XXe siècles : les sources », In Situ [En ligne],

12 | 2009, mis en ligne le 03 novembre 2009, consulté le 14 novembre 2019. URL : http://

journals.openedition.org/insitu/4863 ; DOI : 10.4000/insitu.4863

Ce document a été généré automatiquement le 14 novembre 2019.

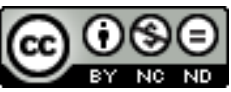

In Situ Revues des patrimoines est mis à disposition selon les termes de la licence Creative Commons Attribution - Pas d'Utilisation Commerciale - Pas de Modification 4.0 International. 


\title{
Les objets mobiliers des XIXe et XXe siècles : les sources
}

\author{
Laurence de Finance
}

\section{Les sources manuscrites}

\section{Les archives nationales}

1 Les documents qui concernent les objets et le mobilier des $\mathrm{XIX}^{\mathrm{e}}$ et $\mathrm{XX}^{\mathrm{e}}$ siècles se trouvent essentiellement dans les séries F 19 (Cultes), F 21 (Beaux-arts), F 15 (Assistance publique), et série $\mathrm{O} 5$ (maison de l'Empereur).

2 Des archives de nombreuses entreprises (série $\mathrm{AQ}$ ) et de fonds d'ateliers sont consultables au Centre des archives du monde du travail, à Roubaix ${ }^{1}$.

\section{Les archives départementales}

Les documents relatifs aux objets et au mobilier des $\mathrm{XIX}^{\mathrm{e}}$ et $\mathrm{XX}^{\mathrm{e}}$ siècles sont essentiellement réunis dans la série $\mathrm{V}$ (cultes). L'entretien des objets du culte et leur renouvellement, les legs et donations sont discutés et précisés dans les délibérations des conseils municipaux. On peut en général y consulter également l'inventaire des biens de la paroisse dressé dans chaque commune en 1905.

\section{Les archives diocésaines}

4 Registres de catholicité (actes de baptême, mariage et sépulture), semaines religieuses et visites pastorales du XIX ${ }^{\mathrm{e}}$ siècle (témoignage de bénédiction d'autels, de verrières, etc.) sont en principe déposés aux Archives départementales, dans un fonds diocésain spécifique. Les documents ayant moins de 100 ans, donc concernant le $\mathrm{XX}^{\mathrm{e}}$ siècle, peuvent être conservés et consultés dans les archives de chaque diocèse. 


\section{La médiathèque de l'Architecture et du Patrimoine (Paris)}

5 L'essentiel des archives conservées concerne des bâtiments et objets protégés au titre des Monuments historiques ${ }^{2}$ : archives de la commission supérieure (aujourd'hui nationale) des Monuments historiques, dossiers de travaux et de restaurations de bâtiments protégés avec délibérations, archives et documents graphiques sur la pose de lambris, vitraux, décor et aménagement de chœur. De nombreuses notes et fonds d'architectes et d'inspecteurs y sont également conservés. À consulter également, l'inventaire du mobilier et œuvres d'art des évêchés et séminaires dressé en 1907-1908 ${ }^{3}$ par l'inspecteur Paul-Frantz Marcou, chargé des objets mobiliers depuis 1893.

Le service des objets mobiliers archive le dossier d'inscription ou de classement au titre des Monuments historiques des objets protégés (arrêtés, photographies), ainsi que les listes de classement par département.

\section{Les archives privées}

7 Celles des artistes (architectes, peintres verriers, peintres, sculpteurs, etc.) et de leurs ateliers (registres de commande, factures, inventaire après décès) ${ }^{4}$, ou celles des commanditaires et collectionneurs fournissent de très précieux renseignements quand elles sont accessibles.

\section{Les musées}

8 Les musées gardent bien souvent dans leurs réserves des fonds d'artistes, dont le contenu n'est pas connu avant qu'une exposition ne les révèle au grand public, comme celle organisée en 2006 au Palais des Beaux-Arts de Lille qui a permis de découvrir la très riche collection de cartons de vitraux des peintres Mottez, Gaudelet, Chérier 5 . Des fonds de sculpteurs contribuent également à la richesse des musées. Si la localisation de certains est évidente comme les fonds Bouchard, Bourdelle, Rodin aux musées parisiens éponymes, d'autres sont plus difficiles à repérer comme les fonds Dalou et Carriès au Petit Palais à Paris, le fonds Injalbert à Béziers ou celui de Pradier au musée d'Art et d'histoire de Genève.

Voir annexe 1 : Archives concernant le mobilier d'église des $\mathrm{XIX}^{\mathrm{e}}$ et $\mathrm{XX}^{\mathrm{e}}$ siècles

\section{Bibliographie}

Devant le nombre d'ouvrages parus sur l'art des $\mathrm{XIX}^{\mathrm{e}}$ et $\mathrm{XX}^{\mathrm{e}}$ siècles, aucune liste ne saurait être exhaustive. À côté des ouvrages généraux, d'autres concernent soit une période bien délimitée, soit une catégorie technique précise, d'autres enfin sont consacrés à un artiste en particulier (monographie, catalogue raisonné, catalogue d'exposition). 
Figure 1

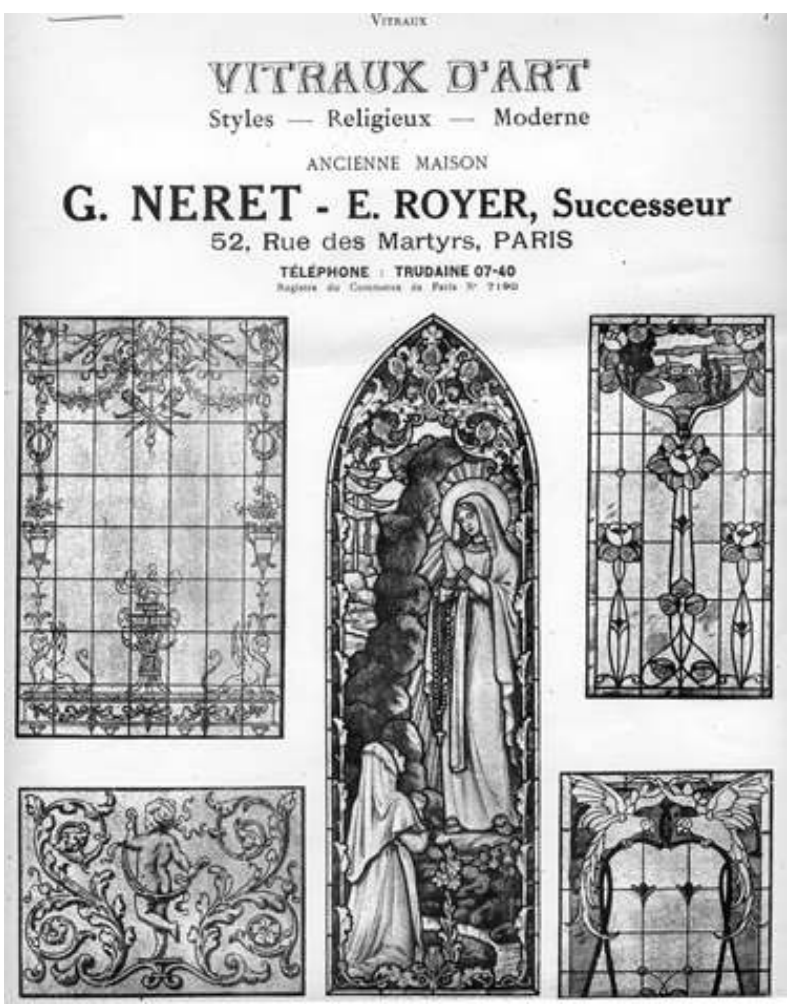

Page publicitaire des peintres verriers Néret et Royer dans le CATALoguE MODĖLE DE L'ARCHITECTE, tome 2 , volume $\mathrm{V}$, section vitraux d'art.

Repro. Laurence de Finance, SDARCHETIS @ MCC.

11 À partir de 1830, de nombreux annuaires de professionnels et entreprises liés au métier de la construction sont publiés annuellement tels les Annuaires Sageret qui fournissent les noms, qualité et adresse des différents artistes et artisans ainsi qu'un aperçu de leur fabrication. Au XXe siècle, l'Annuaire Lahure $(1903,1910)$ et les pages illustrées du Catalogue modèle de l'Architecte (2 tomes, 1913-1916) livrent de nombreux renseignements sur la production des différents corps de métier de la construction. (fig. $\left.n^{\circ} 1\right)$

La bibliographie sur le vitrail des $\mathrm{XIX}^{\mathrm{e}}$ et $\mathrm{XX}^{\mathrm{e}}$ siècles, en annexe, est un exemple de la documentation disponible sur une technique précise à une période donnée. Des renseignements bibliographiques équivalents pourraient être réunis pour chaque catégorie technique : sculpture, peinture, etc.

Voir annexe 2 : Bibliographie des vitraux du XIX ${ }^{e}$ siècle

Voir annexe 3 : Bibliographie des vitraux du $\mathrm{XX}^{\mathrm{e}}$ siècle

\section{Ressources en ligne}

Sur le site du ministère de la Culture et de la Communication, des référentiels, proposant une aide à la recherche et à la connaissance, sont consultables sur le site : Architecture et Patrimoine. (fig. $n^{\circ}$ 2) 

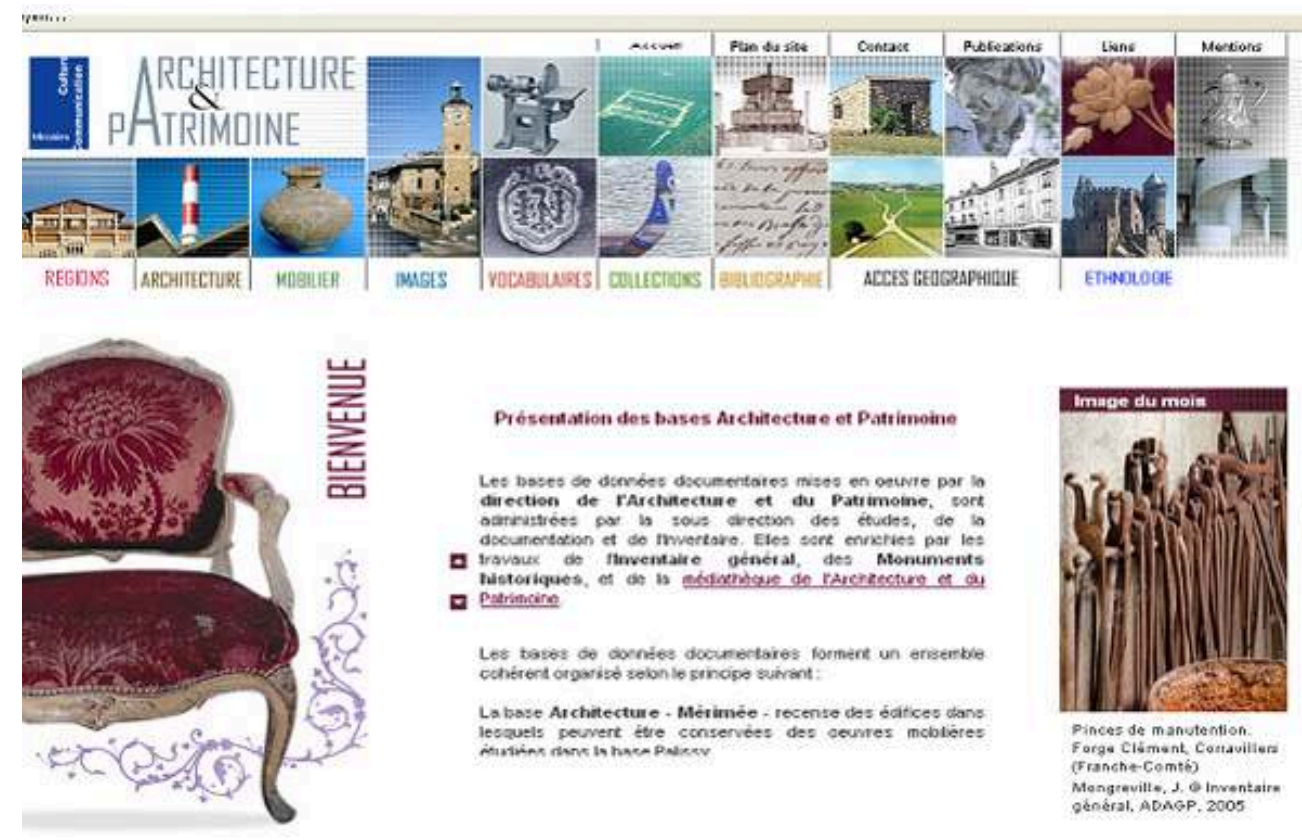

Portail Architecture et patrimoine. Le tableau figurant en annexe 4 donne son mode d'emploi.

Voir annexe 4 : Les bases de données documentaires de l'architecture et du patrimoine

\section{Base auteurs}

Pour accéder à la Base Auteurs: ouvrir l'onglet Vocabulaires puis aller dans Auteurs. Utiliser le formulaire de recherche ou l'interrogation par listes (symboles ou patronymes).

15 Cette base réunit à ce jour près de 6308 notices biographiques d'orfèvres parisiens, actifs de 1875 à 1924 inclus, illustrées de leur poinçon (au 1er septembre 2009, 3980 photos de poinçons sont intégrées, le complément est en cours de validation). Cette base fait suite aux deux volumes du Dictionnaire des poinçons parisiens publiés en 1991 et 1994, concernant les décennies précédentes. Elle est alimentée par la transcription des registres manuscrits de la Garantie de Paris, où les orfèvres sont tenus de déclarer leur métier et de faire enregistrer leurs poinçons.

L'enrichissement de ce corpus se poursuit régulièrement. À terme, plus de 8000 notices biographiques d'orfèvres inscrits à la Garantie de Paris aux XIX et $\mathrm{XX}^{\mathrm{e}}$ siècles seront consultables en ligne, illustrées d'un poinçon de maître, avec possibilité d'actionner des liens vers les notices d'œuvres correspondantes de la base Palissy.

17 La base Auteurs réunit également des notices biographiques sur les peintres verriers français. En septembre 2009, 967 peintres verriers (dont le patronyme commence par des lettres comprises entre $\mathrm{A}$ et $\mathrm{H}$ inclus) ont fait l'objet d'une courte notice permettant de donner les dates d'activité et la localisation de l'atelier ainsi que les départements dans lesquels des verrières de l'artiste ont été recensées. Si certaines de ces verrières sont référencées dans la Base Palissy, un lien donne accès au texte et éventuellement à la photo intégrée dans cette base documentaire. L'enrichissement de ce corpus devrait se poursuivre en 2010. (fig. $\mathbf{n}^{\circ}$ 3) 
Figure 3

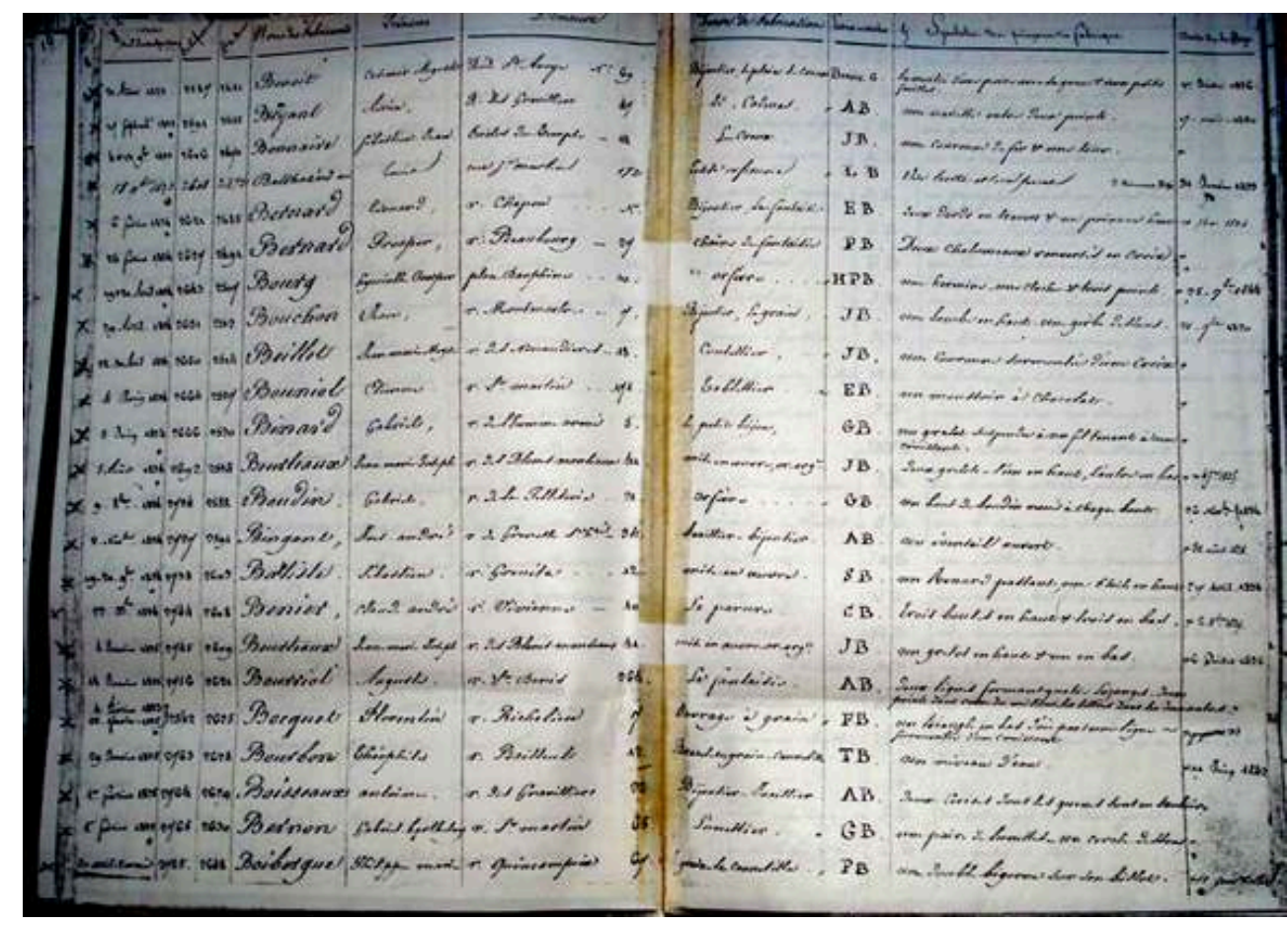

Registre de la Garantie de Paris à partir duquel sont créées les notices des orfèvres de la base Auteurs.

Repro. Laurence de Finance, SDARCHETIS @ MCC.

Voir annexe 5 : Principaux ateliers de peintres verriers des $\mathrm{XIX}^{\mathrm{e}}$ et $\mathrm{XX}^{\mathrm{e}}$ siècles

\section{Base d'iconographie chrétienne}

Pour accéder à la Base d'iconographie chrétienne : ouvrir l'onglet Vocabulaires puis aller dans Iconographie. Utiliser le formulaire de recherche experte.

Cette base regroupe 3538 notices consacrées à l'iconographie issue de l'Ancien et du Nouveau Testament et à celle de la vie des saintes et saints. Cette base iconographique est conçue à partir des intitulés utilisés dans le champ Représentation de la base Palissy. Les illustrations qui y sont présentées permettent d'identifier les scènes et d'en retrouver éventuellement la source graphique ayant inspiré l'artiste. Le répertoire réunit des estampes fréquemment reproduites et donnant lieu à des répliques ou à des copies partielles, inversées ou totales des grands maîtres.

21 Des liens proposés vers des notices d'œuvres des bases Palissy, Mémoire, Joconde, Arcade, Enluminures offrent un large panorama de la documentation réunie à ce jour sur un même thème, traité sur divers supports et à différentes époques. Les illustrations présentées ici montrent que l'auteur de la Visitation d'Allerey-sur-Saône s'est inspiré du tableau de Pierre Mignard dont il a sans doute eu connaissance par la gravure de Fillœul. (fig. $n^{\circ} 4, n^{\circ} 5$ ) 
Figure 4

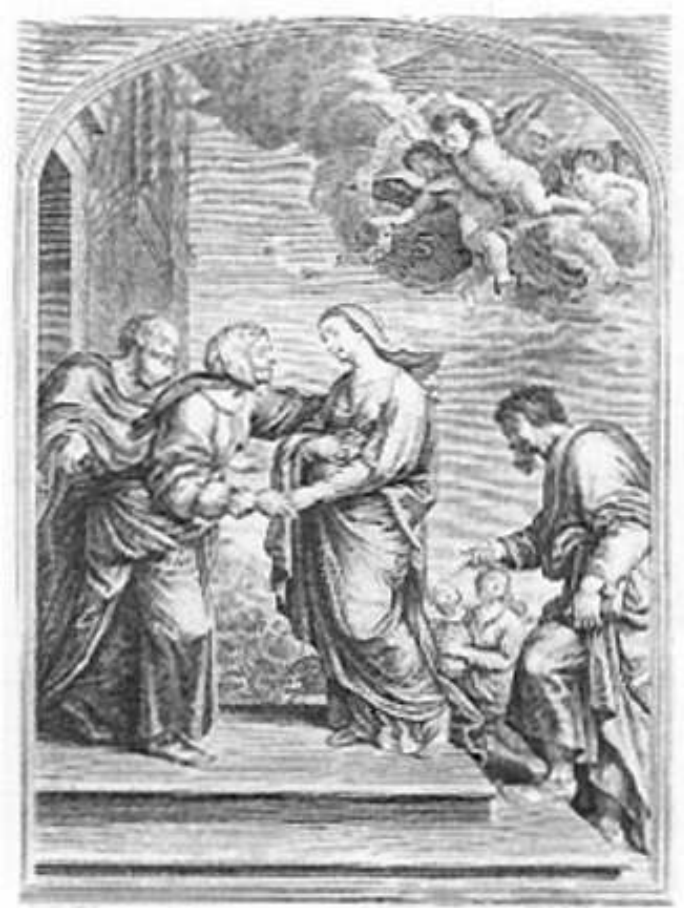

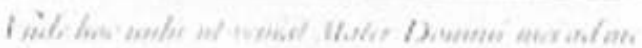

La VISITATION, gravée par Fillœul d'après le tableau de Pierre Mignard. Repro. à partir de AUTOUR DE LA NATIVITÉ dANS LA PEINTURE DES COLLECTIONS ANgEVINES, 1990 
Figure 5

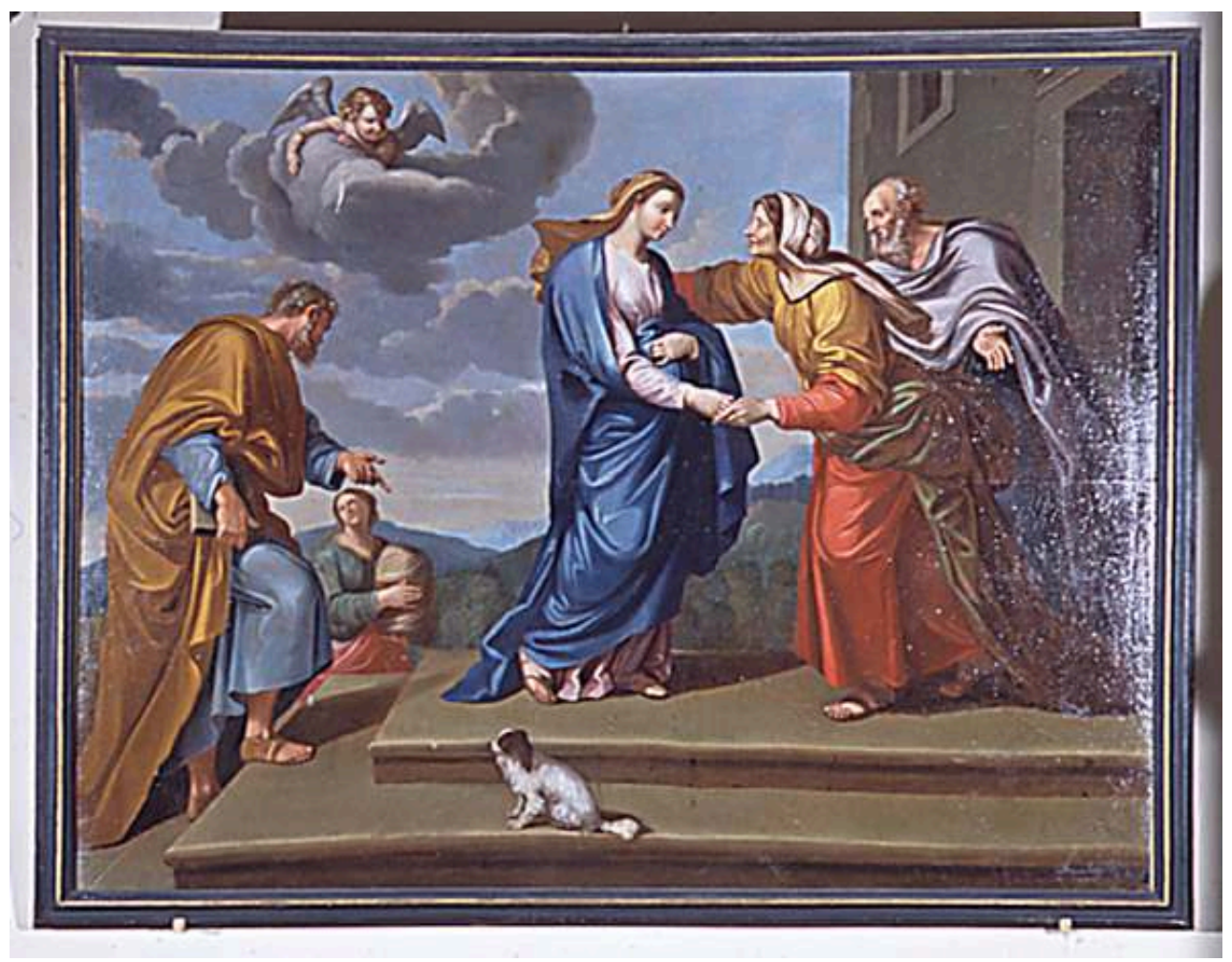

La visıtatıon, église paroissiale d'Allerey-sur-Saône (Saône-et-Loire), copie inversée et interprétée de l'œuvre de Mignard. Phot. M. Rosso.

(c) Inventaire général, Région Bourgogne.

\section{Les catalogues illustrés d'œuvres datées}

Pour accéder aux œuvres datées : choisir l'onglet Collections puis aller dans formes et dates. Choisir le catalogue à consulter. 
Figure 6

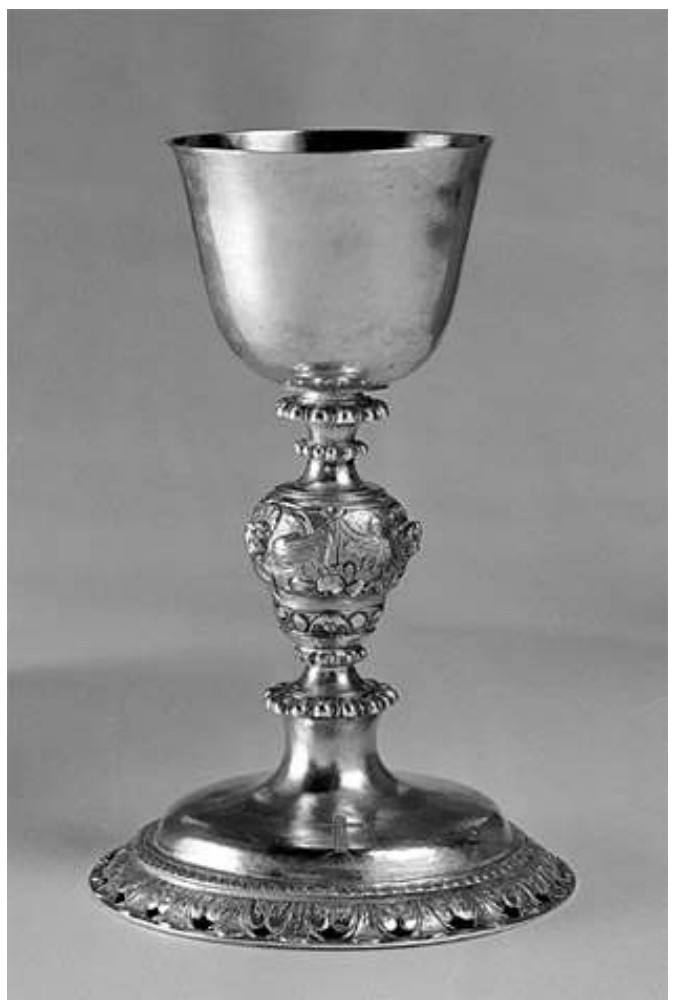

Calice à pied circulaire bordé d'une frise d'acanthes ajourée, selon la mode en vigueur à Paris à partir de 1620. Euvre réalisée en 1623 par l'orfèvre bisontin Pierre de Loisy, conservée à Saint-Lupicin (Jura). Phot. Yves Sancey.

(C) Inventaire général, Région Franche-Comté.

23 Ce répertoire présente l'évolution formelle et stylistique d'un même objet depuis les premiers siècles jusqu'à l'an 2000. À des données historiques, techniques et liturgiques sur l'utilisation de l'objet à travers les siècles ont été jointes des mosaïques d'images permettant de suivre son évolution par quart de siècle du $\mathrm{XVI}^{\mathrm{e}}$ au XXI ${ }^{\mathrm{e}}$ siècle. Trois catalogues sont en ligne, chacun est consacré à un thème particulier : les autels, les calices, les fonts baptismaux. Constitués uniquement d'œuvres datées conservées dans la France entière, ces catalogues sont proposés comme un repère pour dater par comparaison stylistique des œuvres non datées. (fig. $\mathbf{n}^{\circ} \mathbf{6}$ )

\section{Les catalogues commerciaux}




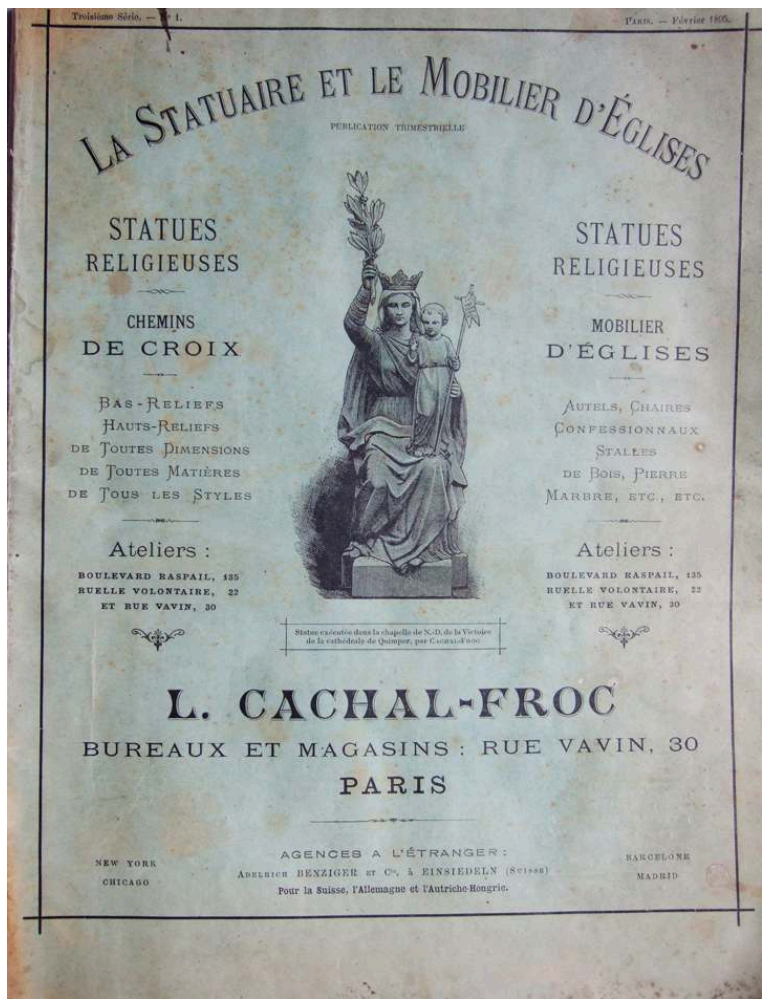

La Statuaire et le mobilier d'églises, couverture du catalogue de la maison Cachal-Froc en 1895.

Repro. Laurence de Finance, SDARCHETIS @ MCC.

Pour accéder aux catalogues commerciaux : ouvrir le portail du ministère de la Culture et de la Communication, consulter, dans les dossiers thématiques, la rubrique de l' Inventaire général du patrimoine culturel. Ouvrir, dans Sources et Ressources, la rubrique Ressources scientifiques. Les documents présentés peuvent être utiles aux professionnels ainsi qu'à toute personne intéressée par le patrimoine religieux: une étude critique porte sur plus de 300 catalogues d'ameublement religieux des $\mathrm{XIX}^{\mathrm{e}}$ et $\mathrm{XX}^{\mathrm{e}}$ siècles, suivie de la liste des entreprises concernées. Toutes les pages de cinq catalogues sont consultables dans leur édition originale illustrée. Ce site est destiné à recevoir d'autres documents, d'autres provenances, afin de faire profiter le plus grand nombre d'une documentation souvent difficile d'accès. Il pourrait par exemple être enrichi par des services régionaux (Inventaire général du patrimoine culturel) détenteurs de catalogues originaux. Des liens vers des notices d'œuvres (base Palissy) issues d'entreprises référencées seront activés afin de relier des œuvres conservées à des fabricants identifiés. (fig. $\mathbf{n}^{\circ}$ 7)

Des informations sur les œuvres présentées aux Salons sont disponibles sur la Base de données ARCHIM.

Conservés au Centre historique des Archives nationales (CP F/21/*7635 à 7668), les 33 albums dits « des Salons » forment un ensemble homogène présentant des œuvres d'art (peintures, sculptures, médailles, pièces d'orfèvrerie, relevés d'architecture) achetées chaque année par le Bureau des Travaux d'art aux artistes exposés au Salon à Paris. Le Salon est organisé par l'administration des Beaux-Arts jusqu'en 1882, puis par la Société des Artistes français (Champs-Élysées). À partir de 1890, un second Salon, agréé par l'État, est organisé par la Société nationale des Beaux-Arts (Champ de Mars). Les 
photographies des œuvres dont l'État fait l'acquisition à ce second Salon sont incluses dans les albums.

Figure 8

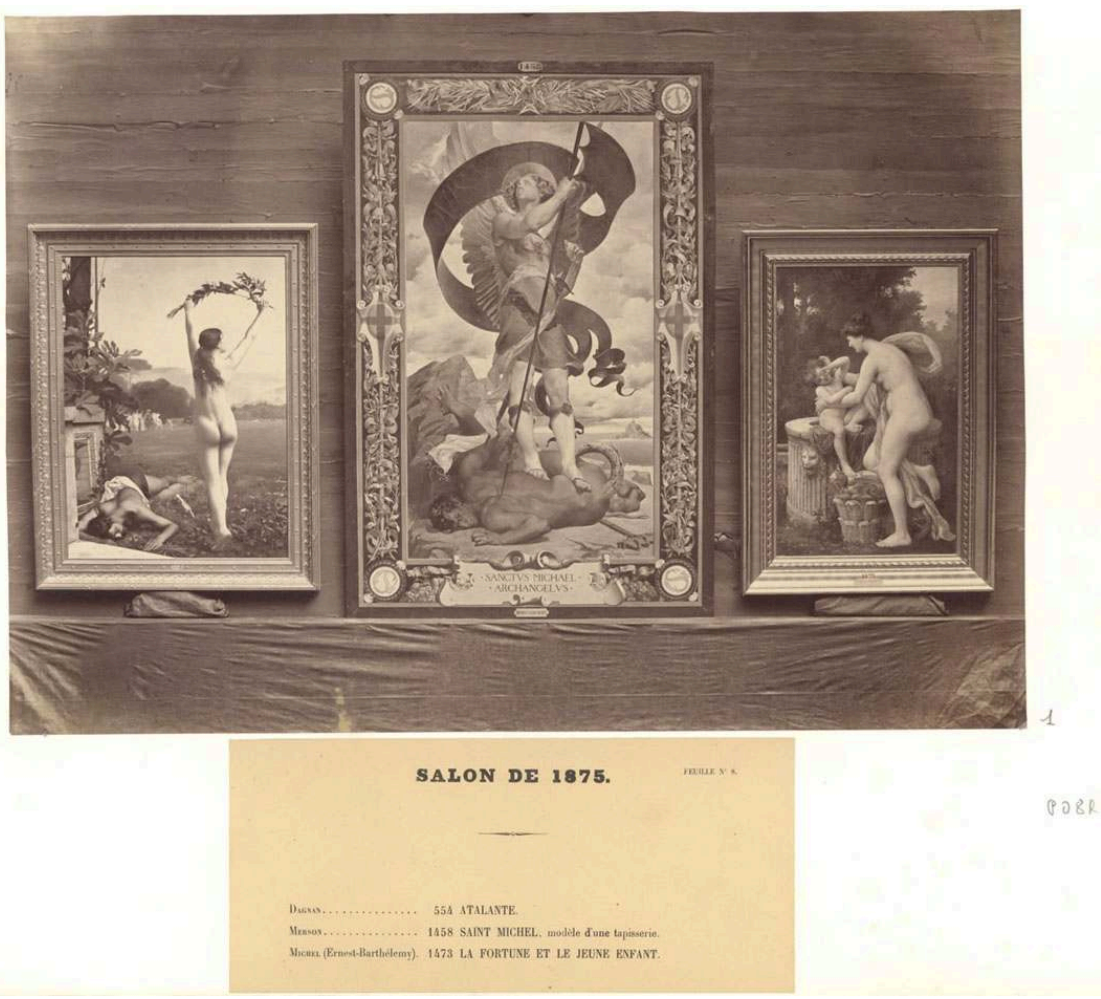

Extrait de la base Archim. Salon de 1875. F21/7645, planche 8.

La Base Arcade retrace, à partir des archives, la genèse et l'histoire des œuvres d'art acquises, commandées ou gérées par l'État et les collectivités territoriales de 1800 à 1939. Elle donne accès aux albums photographiques des Salons de 1864 à 1901. (fig. $\mathrm{n}^{\circ}$ 8)

Figure 9

\section{in Institut national du patrimoine}

Communication présentée lors du séminaire LE PATRIMOINE RELIgIEUX DES XIX ${ }^{\mathbf{E}}$ ET XX $\mathbf{X X}^{\mathbf{E}}$ SIÈCLE qui s'est tenu du 9 au 11 juin 2008 à l'Institut national du patrimoine, avec la participation de la direction de l'Architecture et du patrimoine. 


\section{NOTES}

1. Voir infra l'article de Hottin Christian "Le fonds des maîtres verriers Hebert-Stevens-Bony ", qui montre bien la richesse des fonds d'artistes conservés à Roubaix. Faire le lien svp

2. Sur le patrimoine mobilier classé, lire Kagan Judith, «la loi du 9 décembre 1905 et la conservation du patrimoine mobilier protégé «au titre des monuments historiques». Une genèse. ", Regards sur les églises de France, lieux de culte, lieux de culture, Arles : Actes sud / ACAOAF, 2006, p. 19-46.

3. Sur l'inventaire Marcou voir les précisions données par Judith Kagan, ouvrage cité note 2, 2006, p. 24-25.

4. Voir infra l'article sur Maurice Denis et Henri Carot. Faire le lien svp

5. Voir le catalogue de l'exposition « du dessin au vitrail », organisée par M. Moyne et C. Guillot.

\section{RÉSUMÉS}

L'étude scientifique de tout objet ne peut se concevoir sans une recherche dans les sources documentaires. Qu'il s'agisse d'archives publiques ou privées, de legs faits à des musées ou autres institutions patrimoniales, la quête de renseignements est une nécessité pour suivre l'objet étudié depuis sa création jusqu'à son étude faite in Situ. De nos jours, de nombreuses ressources sont mises en ligne, notamment sur le site du ministère de la culture et de la communication ; accessibles à tous, elles rendent plus aisées la localisation des sources et la consultation de leur contenu. A titre d'exemple, afin de faciliter l'étude du mobilier religieux du XIX ${ }^{\mathrm{e}}$ siècle, dont la production est alors en pleine expansion, des pages illustrées de plusieurs catalogues de fabricants sont aujourd'hui consultables en ligne.

The scientific study of any object requires research in documentary sources. Whether these are private or public archives, legacies left to museums or other heritage institutions, the search for information is a necessity for understanding the life of the object, from its creation to its study in situ. Today, many types of documentary source are available on line, in particular on the website of the French Ministry of Culture. These sources are available to all and easy to consult. The example presented here, concerning the study of religious furnishings of the $19^{\text {th }}$ and $20^{\text {th }}$ centuries, the production of which increased considerably during these centuries, are the pages taken from the catalogues of several manufacturers of these church furnishings, today accessible on line. 


\section{INDEX}

Keywords : moveable objects of the 19th and 20th centuries, moveable objects at the French national archives, moveable objects in French departmental archives, moveable objects in diocesan archives, inventory of moveable objects by P.-F. Marcou, artists' papers, dictionary of Paris gold and silver smiths' hallmarks, the 'auteurs' database of the ministry of culture, commercial catalogues of religious furnishings, moveable objects, online resources, catalogue of dated chalices, catalogue of dated altars, catalogue of dated fonts, salon albums

Mots-clés : les albums des salons, catalogue des fonts baptismaux datés, catalogue des calices datés, catalogue des autels datés, les objets mobiliers aux archives nationales, les objets mobiliers aux archives départementales, les objets mobiliers aux archives diocésaines, inventaire mobilier de P.-F. Marcou, les fonds d'artistes, dictionnaire des poinçons des orfèvres parisiens, base auteurs du ministère de la culture, les catalogues commerciaux de mobilier religieux, objets mobiliers : ressources en ligne

\section{AUTEUR}

\section{LAURENCE DE FINANCE}

Conservateur en chef du patrimoine, sous-direction de l'archéologie, de l'ethnologie, de l'inventaire et du système d'information, direction de l'architecture et du patrimoine. laurence.de-finance@culture.gouv.fr. ldefinance@citechaillot.fr 